Turbulence correlation properties measured with ultrafast sweeping reflectometry on Tore Supra

This content has been downloaded from IOPscience. Please scroll down to see the full text.

View the table of contents for this issue, or go to the journal homepage for more

Download details:

IP Address: 157.193.65.248

This content was downloaded on 03/12/2013 at 11:38

Please note that terms and conditions apply. 


\title{
Turbulence correlation properties measured with ultrafast sweeping reflectometry on Tore Supra
}

\author{
G Hornung ${ }^{1}$, F Clairet ${ }^{1}$, G L Falchetto ${ }^{1}$, R Sabot ${ }^{1}$, H Arnichand $^{1}$ \\ and $L$ Vermare ${ }^{2}$ \\ ${ }^{1}$ CEA, IRFM, F-13108 Saint-Paul-lez-Durance, France \\ ${ }^{2}$ Laboratoire de Physique des Plasmas, Ecole Polytechnique, CNRS, 91128 Palaiseau Cedex, France \\ E-mail: gregoire.hornung@gmail.com
}

Received 29 March 2013, in final form 16 September 2013

Published 20 November 2013

Online at stacks.iop.org/PPCF/55/125013

\begin{abstract}
The ultrafast sweep reflectometry technique is used to investigate plasma turbulence on Tore Supra. Thanks to the X-mode polarization, the plasma density and fluctuations over the whole tokamak radius, from the far scrape-off layer (SOL) to the core can be measured.

Cross-correlation analysis is applied to all the signal components to estimate the fluctuation correlation length radial profile. The correlation length decreases from the plasma centre to the last closed flux surface and grows again into the far SOL.
\end{abstract}

(Some figures may appear in colour only in the online journal)

\section{Introduction}

Anomalous transport seriously affects the performance of nuclear fusion reactors. Reducing anomalous transport requires understanding the properties of the underlying turbulent structures. Reflectometry has long been used to measure turbulence fluctuations and correlation lengths mostly using fixed frequency systems. The conventional method of two-frequency reflectometry (radial correlation reflectometry) uses two separate microwave systems with different frequencies [1-8].

Here, we present turbulence correlation properties measured using the frequency modulated continuous wave (FMCW) reflectometry as a continuous sweep conveniently provides measurements from the edge of the plasma to the centre. Such a technique can be used as far as the turbulence is locally frozen during the sweeping time, which allows us to obtain a valid estimate of the correlation lengths. A comparable study was carried out by Kurzan et al in 2000 [9]; here, the study is performed with a recently improved reflectometer system using faster frequency sweeps $(2 \mu \mathrm{s})$ and heterodyne detection, where phase and amplitude of the reflected signals can be measured separately [10]. Thanks to the X-mode polarization, the temporal and spatial turbulence correlation properties of the raw fluctuating data have been analysed from the far scrape-off layer (SOL) to the core plasma region in Tore Supra ohmic discharges.

The sweeping reflectometer setup is detailed in section 2 . Section 3 introduces the correlation analysis techniques which have been applied to the reflectometer signals to extract the fluctuation coherency, correlation lengths and times as well as radial velocities. Plasma conditions and results on the above quantities are presented in section 4. Finally, the results are reviewed and discussed in section 5.

\section{Tore Supra reflectometers}

The ultrafast sweep upper cut-off X-mode heterodyne reflectometer used in this work operates in the $\mathrm{V}$ - and W-bands with frequency range $50-75 \mathrm{GHz}$ and $75-110 \mathrm{GHz}$, respectively. Each frequency band is swept in $2 \mu$ s with a $1 \mu \mathrm{s}$ dead time, giving a temporal resolution of $3 \mu \mathrm{s}$. Both reflectometers are swept simultaneously thus there is a time lapse of $2 \mu \mathrm{s}$ between the end of the V-band and the beginning of the $\mathrm{W}$-band (i.e. the $75 \mathrm{GHz}$ of the V-band is emitted $2 \mu \mathrm{s}$ after the $75 \mathrm{GHz}$ emitted by the W-band). Several bursts of sweeps can be performed during the plasma discharge with a maximum of 10.000 sweeps due to the memory storage limit of the acquisition module. Each reflectometer signal is acquired 


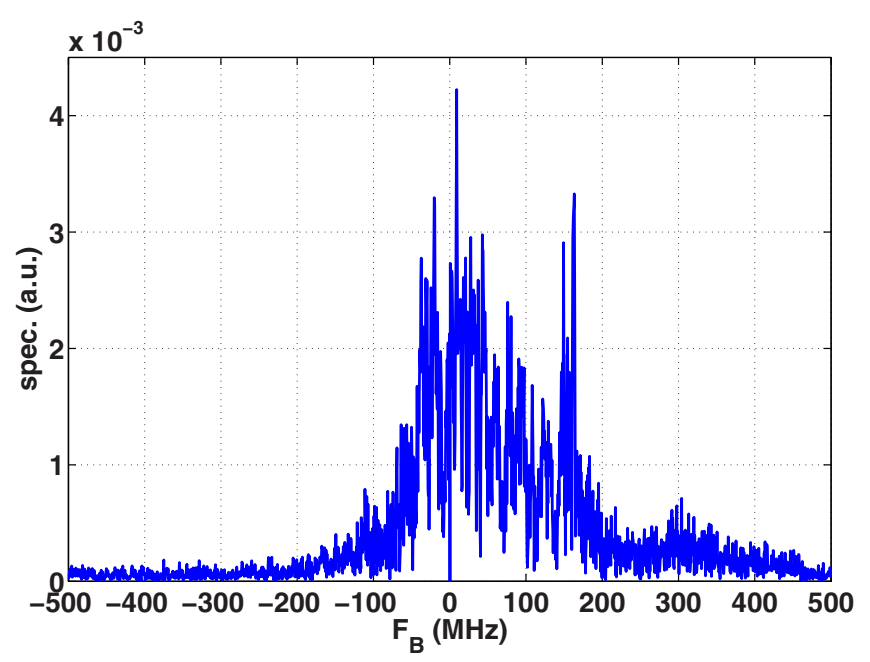

Figure 1. Beat frequency spectrum computed for the discharge \#47475.

with 2000 points at $1 \mathrm{Gs} \mathrm{s}^{-1}$ sampling rate. The reflectometer is located at the outboard midplane of Tore Supra $1 \mathrm{~m}$ away from the SOL plasma. More details on the reflectometer are presented in [11].

The probing wave propagates into the plasma up to a critical plasma layer, the cut-off layer. The frequency sweeping provides a radial scan of the plasma from the edge to the centre. At the cut-off layer, the probing wave is reflected and the fluctuations in the reflected signal are induced by electron density fluctuations.

Data obtained with a fixed frequency reflectometer [12] are also used in the following. The fixed frequency reflectometer operates in the D band $(105-150 \mathrm{GHz})$ and probes the plasma in the equatorial plane. The data acquisition frequency is $1 \mathrm{MHz}$ leading to $1 \mu$ s temporal resolution.

The complex signal measured and recorded by the reflectometers at time $t$ is expressed as:

$$
S(F, t)=A(F, t) \mathrm{e}^{\mathrm{i} \phi(F, t)} .
$$

$A$ and $\phi$ are the amplitude and phase of the signal, respectively. $F$ is the probing signal frequency.

\section{Signal analysis}

The sweep systems offer the great advantage of working with plasma reflected signals from which the undesired echoes and especially the multi-reflections have been filtered out. As shown in figure 1, the measured beat frequencies vary approximately over a range of $500 \mathrm{MHz}$. In order to isolate the signal reflected by the plasma a sliding filtering technique [11] is used, with a sliding bandwidth of $100 \mathrm{MHz}$ around the beat frequency evolution.

The electron density profile is reconstructed for each frequency sweep according to the Bottollier-Curtet recursive algorithm [13,14]. The radial dependence of the cut-off frequencies is given from the mean density profile which is obtained by averaging over the $\mathrm{N}$ sweeps acquired during a single burst. Then, the frequency dependence of the reflectometer signal is replaced by the radial dependence. The fluctuating quantities are calculated from the temporal departures of the mean value:

$$
\delta X(r, t)=X(r, t)-\overline{X(r)},
$$

$X(r t)$ denote any components of the reflected signals, namely either the full complex signal, the real or imaginary part, the phase or the amplitude. Quantitative properties of the signal fluctuations are extracted through the cross-correlation analysis. The cross-correlation function (CCF) evaluates the similarity between data measured at different positions and times. The $\operatorname{CCF}(r, \Delta r, \Delta t)$ computed on two time series $X(r, t)$ and $X(r+\Delta r, t+\Delta t)$ measured at different positions reads:

$$
\begin{aligned}
& \operatorname{CCF}(r, \Delta r, \Delta t) \\
& =\frac{\overline{(X(r, t)-\overline{X(r)})(X(r+\Delta r, t+\Delta t)-\overline{X(r+\Delta r)})}}{\sigma_{X(r)} \sigma_{X(r+\Delta r)}},
\end{aligned}
$$

$\overline{X(r)}=\sum_{i=1}^{N} X(r)_{i} / N$ and $\sigma_{X(r)}=\left(\sum_{i=1}^{N}\left(X(r)_{i}-\right.\right.$ $\left.\bar{X})^{2} / N\right)^{1 / 2}$ denote the mean and standard deviation performed over the time series of length $\mathrm{N}$ (number of frequency sweeps) measured at the radial position $r$. On the discharges presented in this paper, the time average is performed over $30 \mathrm{~ms}$ $\left(\# 47537, N=10^{4}\right), 15 \mathrm{~ms}\left(\# 47475, N=5 \times 10^{3}\right)$ and $9 \mathrm{~ms}$ $\left(\# 47170, N=3 \times 10^{3}\right)$.

The CCF is meaningful if the fluctuation time scale is much shorter than the time series length. The CCF computed for $\Delta r=0$ is the autocorrelation function (ACF). The correlation time $t_{\text {corr }}$ is defined as the ACF full width at half maximum. Reliable correlation times can be obtained only if there are at least 3 experimental points on the ACF above 0.5 . Given that the time resolution is $3 \mu \mathrm{s}$, the lowest accessible correlation time is thus $t_{\text {corr }}=6 \mu \mathrm{s}$.

The radial correlation length is defined as the $\mathrm{CCF}$ full width at half maximum for $\Delta t=0$. In practice, the correlation time and length are calculated by linearly interpolating the CCF around 0.5 .

If $\mathrm{X}$ is a complex variable, it is more convenient to work with the Fourier transform of the $\operatorname{CCF}(r, \Delta r, \Delta t)$ called coherency

$$
\gamma(r, \Delta r, F)=\frac{\left\langle P_{X_{r} X_{r+\Delta r}}\right\rangle}{\left(\left\langle P_{X_{r} X_{r}}\right\rangle\left\langle P_{X_{r+\Delta r} X_{r+\Delta r}}\right\rangle\right)^{1 / 2}} .
$$

Here $P_{X_{r} X_{r+\Delta r}}, P_{X_{r} X_{r}}$ and $P_{X_{r+\Delta r} X_{r+\Delta r}}$ are the cross and auto power spectra computed on the time series $X(r, t)$ and $X(r+\Delta r, t) .\langle\cdot\rangle$ denotes the ensemble average. In the results presented here, 40 ensembles averages are performed. The coherency gives information on the correlation of each frequency included in the time series. Consequently, a radial coherence length defined as the coherency full width at half maximum can be calculated for each frequency. To avoid any confusion, the subscripts coh and corr are used when we refer to a quantity computed with the coherency and the $\mathrm{CCF}$, respectively.

Figure 2(a) shows an example of the coherency computed on the complex signal. On this example the coherence lengths are larger at low frequencies and decrease slightly when the 

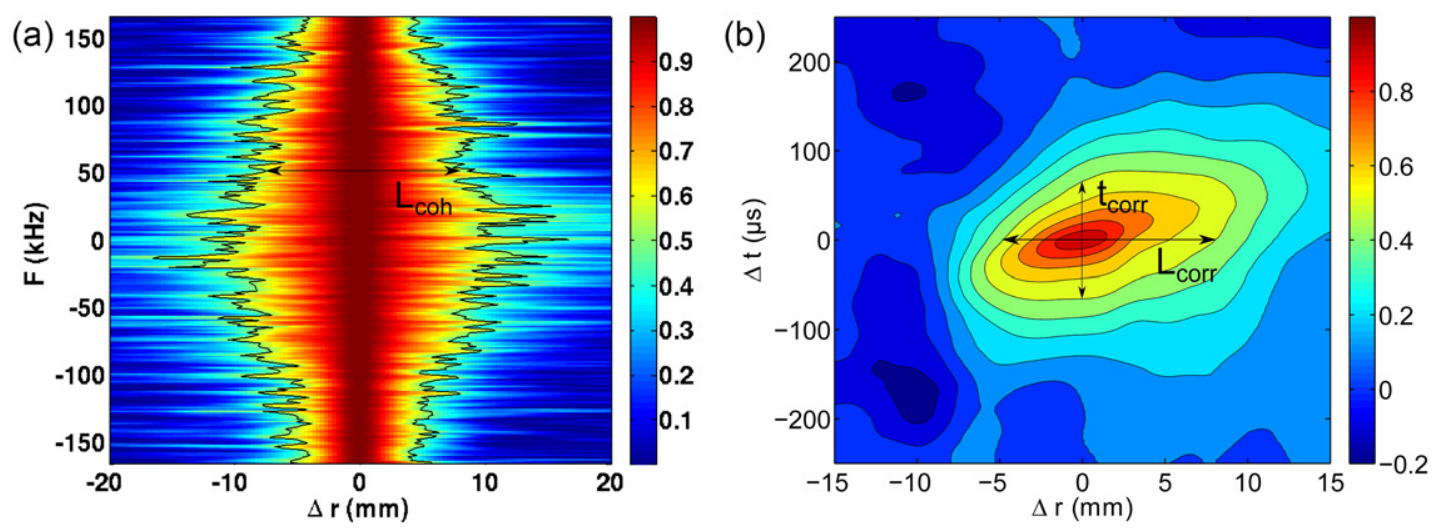

Figure 2. (a) Contour plot of the coherency computed on the complex signal in the core plasma $(\rho \sim 0.4)$ of the Tore Supra ohmic shot \#47475. (b) Contour plot of the amplitude cross-correlation function, for Tore Supra ohmic shot \#47170 measured $7 \mathrm{~cm}$ outside the last closed flux surface (LCFS). The coherence length $L_{\text {coh }}$, correlation time $t_{\text {corr }}$ and length $L_{\text {corr }}$ are represented by arrows.

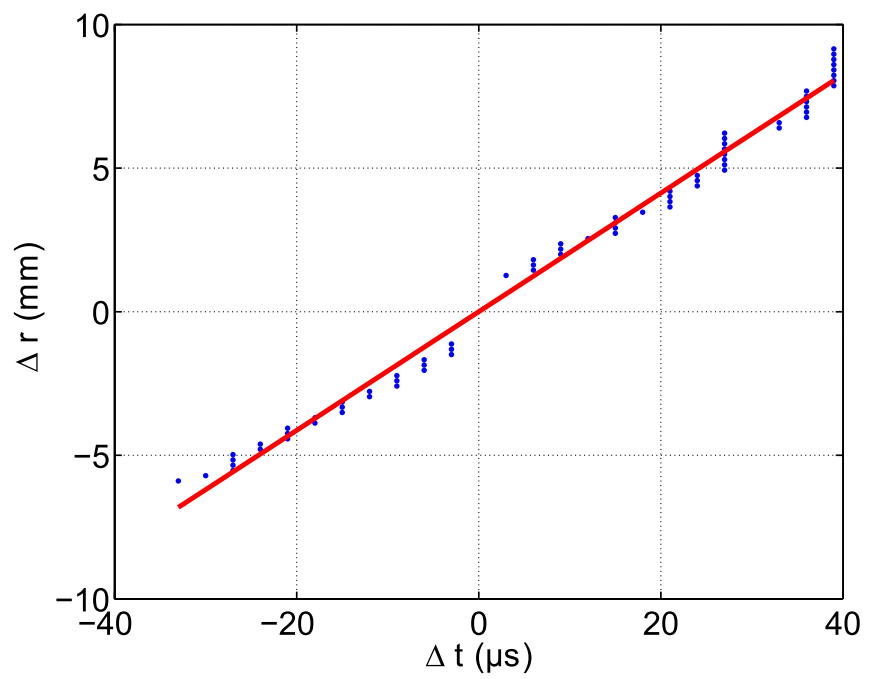

Figure 3. Position of the time lags (blue dots) where the amplitude CCF peaks, for Tore Supra shot \#47170 measured $7 \mathrm{~cm}$ outside the LCFS. Only time lags verifying $\Delta t \neq 0$ and $\operatorname{CCF}(r, \Delta t, \Delta r) \geqslant 0.5$ are kept. The red line is the linear fit from which the radial velocity is estimated.

frequency increases. Figure $2(b)$ shows an example of the CCF computed on the signal amplitude.

In this example, the CCF contour levels are positively tilted in the $(\Delta r, \Delta t)$ plane. This tilt is a consequence of radially outward propagating fluctuations. The time lag $\Delta t$ for which the CCF peaks, satisfying $\Delta t \neq 0$ and $\operatorname{CCF}(r, \Delta t, \Delta r) \geqslant 0.5$, is identified. If at least 10 experimental points fulfill the previous condition, a linear fit of the curve $\Delta r=V_{\text {corr }} \Delta t$ is performed to obtain the radial velocity $V_{\text {corr }}$. Figure 3 illustrates the procedure used to estimate the radial velocity on the amplitude data.

In the following the coherence analysis has been applied to the full complex signal whereas correlation analysis has been applied to the amplitude $A$, phase $\phi$ and the real part of the reflectometer signal $A \cos (\phi)$ (in the literature often referred to as the homodyne signal $[15,16])$. Separate analysis of the amplitude and the phase has been performed as they account for different plasma fluctuation properties. The amplitude better accounts for the 2D (two dimensional), i.e. poloidal and toroidal, mirror reflecting effects, while the phase accounts for the density profile deformation and mostly refers to a $1 D$ radial effect. The complex and homodyne signals are sort of mixes of both phase and amplitude.

\section{Application to Tore Supra ohmic discharges}

One of the great advantages of the X-mode polarization sweeping reflectometry is its capability to cover the plasma from the very far SOL to the core continuously. A snapshot of the entire plasma profile is thus rapidly recovered. However, the accessibility of our V- and W-band reflectometers to the plasma centre is limited to low densities $\left(<3 \times 10^{19} \mathrm{~m}^{-3}\right)$ and low magnetic field $\left(B_{0} \approx 3 \mathrm{~T}\right)$. An extension of the frequency range is underway and will provide access to the core for almost all plasma conditions.

Tore Supra is a circular section limiter tokamak with major radius $R=2.38 \mathrm{~m}$ and minor radius $a=0.72 \mathrm{~m}$. In this paper, correlation measurements obtained on Tore Supra ohmic discharges are presented. First, we show how the fluctuation length profile can be computed over the whole radius. It is then highlighted how magnetohydrodynamic (MHD) activity impacts the determination of the coherence lengths. Finally, the correlation properties are analysed in detail in the edge and SOL plasma, over a discharge which provides better statistics.

In this paper, core $(\rho<0.7)$ and edge $(0.7 \leqslant \rho<1)$ refer to closed magnetic field lines regions, whereas SOL $(\rho \geqslant 1)$ refers to the region outside the last closed flux surface (LCFS). The radial position is labelled by the normalized toroidal flux coordinate $\rho$ for data mostly obtained in the closed magnetic field lines region whereas the distance to the LCFS $d_{\text {LCFS }}$ is used in the SOL.

\subsection{Radial correlation length profile}

Tore Supra shot \#47475 is a low magnetic field ohmic discharge. In these conditions, the accessibility of the reflectometer measurement can cover the plasma from about $\rho \approx 1.2$ to around $\rho \approx 0.3$ and provide a complete density profile from the far SOL to the core region (figure 4(a)). The density fluctuation profile is calculated according to [17] from 

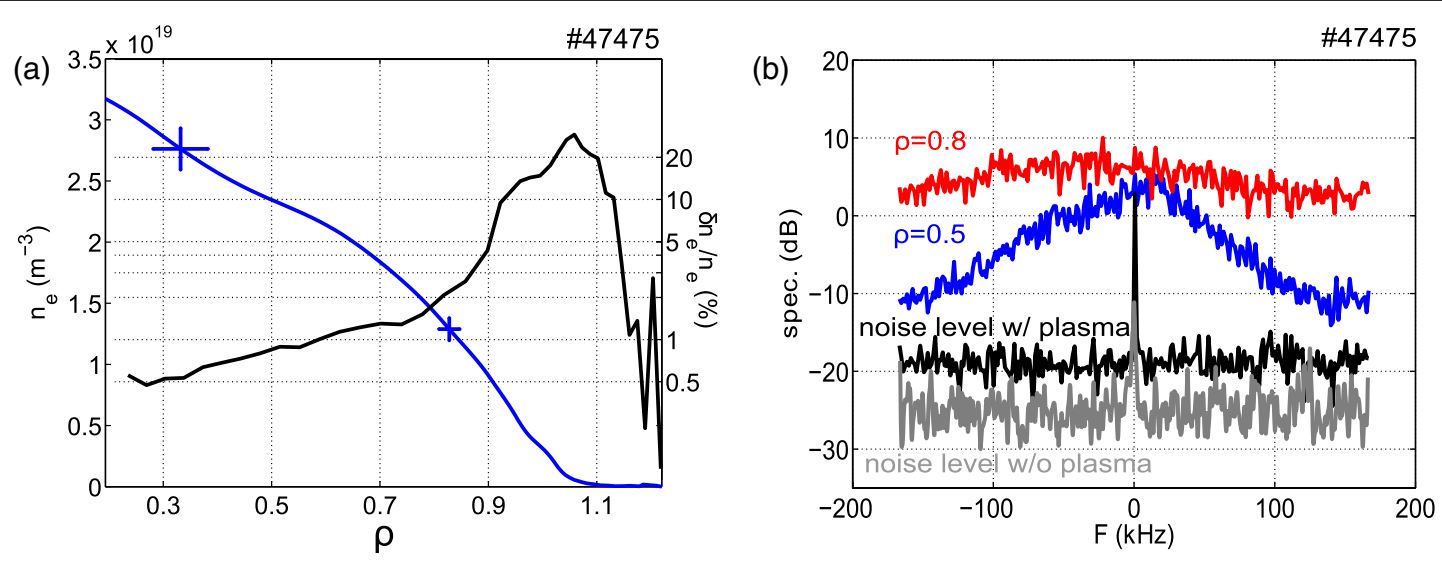

Figure 4. (a) Mean electron density profile. The fluctuation level is superimposed in full black line. The uncertainties, quantified by the standard deviation, are shown for two radial positions. (b) Complex power spectra computed at $\rho=0.5$ (blue curve) and $\rho=0.8$ (red curve). The spectral characteristics of the noise measured with (black curve) and without (grey curve) plasma are also shown.

the radial wavenumber spectra of the radial phase fluctuations integrated between 2 and $10 \mathrm{~cm}^{-1}$. Figure $4(b)$ shows the complex signal power spectra at two different radial positions. In the core plasma at $\rho=0.5$, the spectrum is dominated by frequencies in the range $-50 \mathrm{kHz} \leqslant F \leqslant 100 \mathrm{kHz}$ and falls rapidly for higher frequencies. In the edge plasma, at $\rho=0.8$, the spectrum is flatter. The flattening of the power spectrum might be related to the increase of the fluctuation level, which raises from around $\delta n_{\mathrm{e}} / n_{\mathrm{e}} \approx 0.8 \%$ at $\rho=0.5$ to about $\delta n_{\mathrm{e}} / n_{\mathrm{e}} \approx 2 \%$ at $\rho=0.8$ (figure $4(a)$ ). The spectral characteristics of the noise have been calculated as follows: the signals were bandpass filtered to eliminate the plasma beat frequencies $([-300,-200] \mathrm{MHz}$, see figure 1) during the plasma discharge and to isolate the backwall echoes $([200,300] \mathrm{MHz})$ for the signals measured without plasma. The signal to noise ratio is larger than $20 \mathrm{~dB}$. However, a detailed analysis of the frequency spectra is out of the scope of the present communication and will be reported elsewhere.

5000 frequency sweeps were performed during a burst. During the acquisition, the mean electron density $\left\langle n_{\mathrm{e}}\right\rangle=$ $1.98 \times 10^{19} \mathrm{~m}^{-3}$, the plasma current $I_{\mathrm{p}}=0.7 \mathrm{MA}$ and the magnetic field $B_{0}=3.08 \mathrm{~T}$ were kept constant. Figure 5 shows the radial and temporal dependence of the normalized $A, \phi$ and $A \cos (\phi)$ signals. The signals have been normalized by subtracting the mean value and dividing by the standard deviation to weight the different plasma regions consistently. For this discharge, the connection between the V-and W-band signals occurs at about $\rho=0.9$.

In the far SOL the data show large turbulent structures propagating outwards which are better seen on the amplitude rather than on the phase signal. On the opposite, in the plasma core an MHD tearing mode (the central $q=1$ rational surface is hardly covered by the reflectometer) has a stronger influence on the phase. The effect of MHD activity will be discussed more precisely in the following relating to another plasma discharge.

Figure 6 shows a comparison of the radial evolution of the coherence and correlation lengths from the far SOL to the core plasma. The coherence lengths are measured on the full complex signal whereas the correlation lengths are measured on the amplitude phase and real part of the signal.
Both coherence and correlation lengths decrease from the centimetre range in the core plasma to millimetric at the LCFS and increase again in the SOL plasma. It is worth noting that the continuity between the reflectometer signals is well recovered. The frequency dependence of the coherence lengths is depicted on figure 6(a). In between $\rho=0.4$ and $\rho=1.1$, no difference is observed on the coherence length profiles for any frequency component. For $\rho \leqslant 0.4$, the coherence lengths associated to low frequency components are clearly larger than the coherence lengths associated to high frequencies. Figure $6(b)$ shows the coherence lengths averaged on three distinct frequency intervals: $|F|=[0: 5] \mathrm{kHz}$, $|F|=] 5: 50]$ and $|F|=] 50: 166] \mathrm{kHz}$. The radial profiles of the coherence and correlation length (figure 6(c)) are in close agreement for $\rho>0.4$, whereas they reflect the presence of MHD modes in the inner region. In the latter region, the coherence analysis is more appropriate allowing to separate the contribution of the different frequency components, as it will be further investigated in the following subsection. In the outer core, edge and SOL plasmas, coherence or correlation methods can be equivalently used for the estimate of the fluctuation characteristic scales.

A detailed analysis of the edge region is presented in section 4.3 taking advantage of a discharge where larger statistics and a comparison with fixed frequency reflectometer data are available. In the above an implicit assumption of locally frozen turbulence was used, which will also be proven on that same shot.

\subsection{Effect of MHD activity on the correlation lengths}

Tore Supra shot \#47537 is a low density ohmic discharge. 10.000 reflectometer sweeps were performed corresponding to $30 \mathrm{~ms}$ acquisition. During the sweep, the mean electron density $\left\langle n_{\mathrm{e}}\right\rangle=6.3 \times 10^{18} \mathrm{~m}^{-3}$, the plasma current $I_{\mathrm{p}}=$ 0.6 MA and the magnetic field $B_{0}=3.38 \mathrm{~T}$ were kept constant. Figure 7 shows the radial dependence and temporal evolution of the normalized $A, \phi$ and $A \cos (\phi)$ signals.

The core plasma, is often characterized by a substantial MHD activity related to tearing modes which is well recorded by the reflected signals $[18,19]$. The $q$ profile computed 

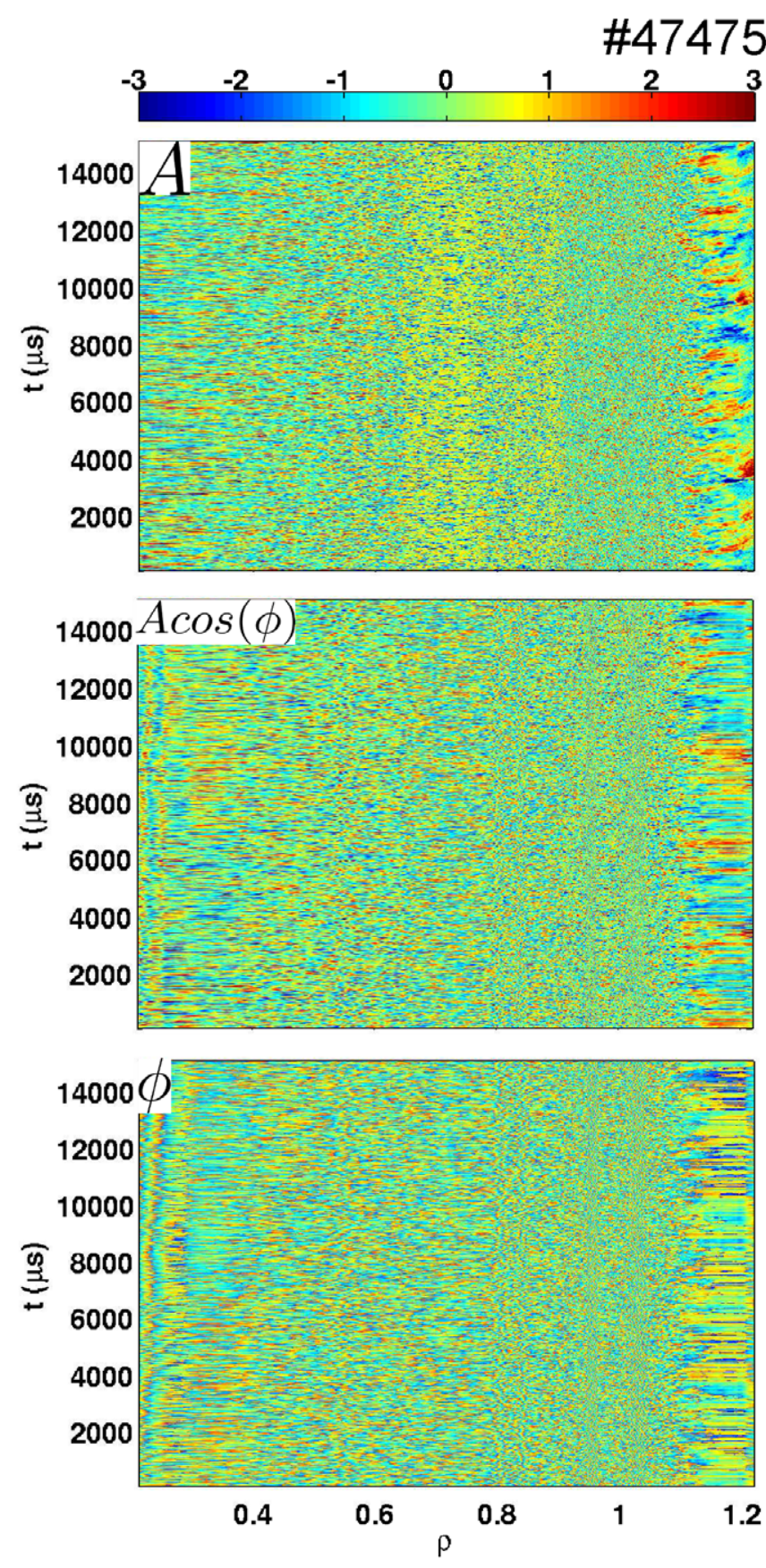

Figure 5. Normalized amplitude (top), real (middle) and phase (bottom) signals with respect to the normalized radial position and time.

with the CRONOS integrated modelling suite code [20] has been superimposed to locate rational surfaces so to give a complementary and necessary information about the position of rational surfaces. It is essential for their identification and the understanding of the perturbed reflectometry data.

The data around the $q=1$ rational surface exhibit a complex radial and temporal evolution. The fast oscillating tearing modes at $2 \mathrm{kHz}$ are clearly visible on both sides of the plasma centre between 10 and $15 \mathrm{~ms}$ with an additional sawtooth crash at about $15 \mathrm{~ms}$. A first sawtooth crash also occurred at the very beginning of the data acquisition, consistently to the ECE signal from which a sawtooth period of approximately $15 \mathrm{~ms}$ is obtained. Moreover, the sawtooth crashes locally trigger an MHD activity at the neighbouring rational surfaces $q=3 / 2$ and $q=2$ as previously observed [21].

While looking at the effects of the sawtooth crash on the coherence lengths, the signal has been analysed over two different time windows, just before $\left(t_{1}=[12: 15] \mathrm{ms}\right)$ and right after the crash $\left(t_{2}=[15: 18] \mathrm{ms}\right)$. The coherence length contour plots (figures $10(a)$ and $(b)$ ) are zoomed between -50 and $50 \mathrm{kHz}$ where most of the differences between the two cases occur. Before the crash, the low frequency $(|F| \sim 0$ $5 \mathrm{kHz}$ ) coherence lengths are particularly large in between $q=1$ and $q=3 / 2$ and outside $q=2$. The coherence lengths associated to higher frequencies are generally lower, apart from those associated to frequencies $|F| \sim 25-50 \mathrm{kHz}$ which reach up to $13 \mathrm{~cm}$ around $q=1$. Right after the crash, the coherence lengths associated to $|F| \sim 25-50 \mathrm{kHz}$ and measured around $q=1$ are strikingly reduced. This reduction is also observed on the $|F| \sim 0-5 \mathrm{kHz}$ coherence lengths measured in between $q=1$ and $q=3 / 2$ and outside $q=2$. Figure 10 shows the coherence length profiles averaged over two-frequency intervals, $|F|=[0: 5] \mathrm{kHz}$ and $|F|=] 5: 166] \mathrm{kHz}$ confirming that the coherence lengths computed before the crash are mainly larger than those computed after the crash. Note that after the crash, the correlation length profiles are similar for all frequencies.

A systematic decrease is observed around $\rho \approx 0.46$, corresponding roughly to the position of $q=2$ rational surface which suggests an interplay with turbulent modes.

\subsection{Edge and SOL regions}

In this subsection, a special attention is paid to the edge and SOL region turbulence properties. This is done by analysing data from the Tore Supra ohmic discharge \#47170. The plasma lasted $15 \mathrm{~s}$ and was in steady state from $t=4$ to $t=12 \mathrm{~s}$. During the steady state operation, the mean electron density $\left\langle n_{\mathrm{e}}\right\rangle=2.1 \times 10^{19} \mathrm{~m}^{-3}$, the plasma current $I_{\mathrm{p}}=1 \mathrm{MA}$ and the magnetic field $B_{0}=3.7 \mathrm{~T}$ did not vary more than $5 \%$. Three successive reflectometer bursts were realized at time $t=5,7.5$ and $10 \mathrm{~s}$ which can thus provide an interesting set of separate and independent measurements for the statistical analysis. Figure 9 shows the mean electron density profile.

The fluctuation profile computed as in [17] is also shown and will be used for discussion. Each burst has 3000 sweeps which corresponds to $9 \mathrm{~ms}$ of measurement time. For each burst at a given radial position, the CCF are independently computed on the $9 \mathrm{~ms}$ time series. Therefore, three $L_{\text {corr }}, t_{\text {corr }}$ and $V_{\text {corr }}$ measurements are available to quantify the variation of the results over the plasma radius.

In order to study the correlation time in the edge plasma, first the sweeping reflectometer measurements are benchmarked against a fixed frequency system operating at $1 \mathrm{MHz}$. Figure 10(a) shows that the RMS level of the phase fluctuations obtained with the sweeping system $\left(\sigma_{\phi}=\right.$ $2.2 \mathrm{rad}$ ) is larger than that with the fixed frequency system $\left(\sigma_{\phi}=0.26 \mathrm{rad}\right)$. A frequency jitter of about $40 \mathrm{MHz}$ occurring between sucessive sweeps was measured in absence 

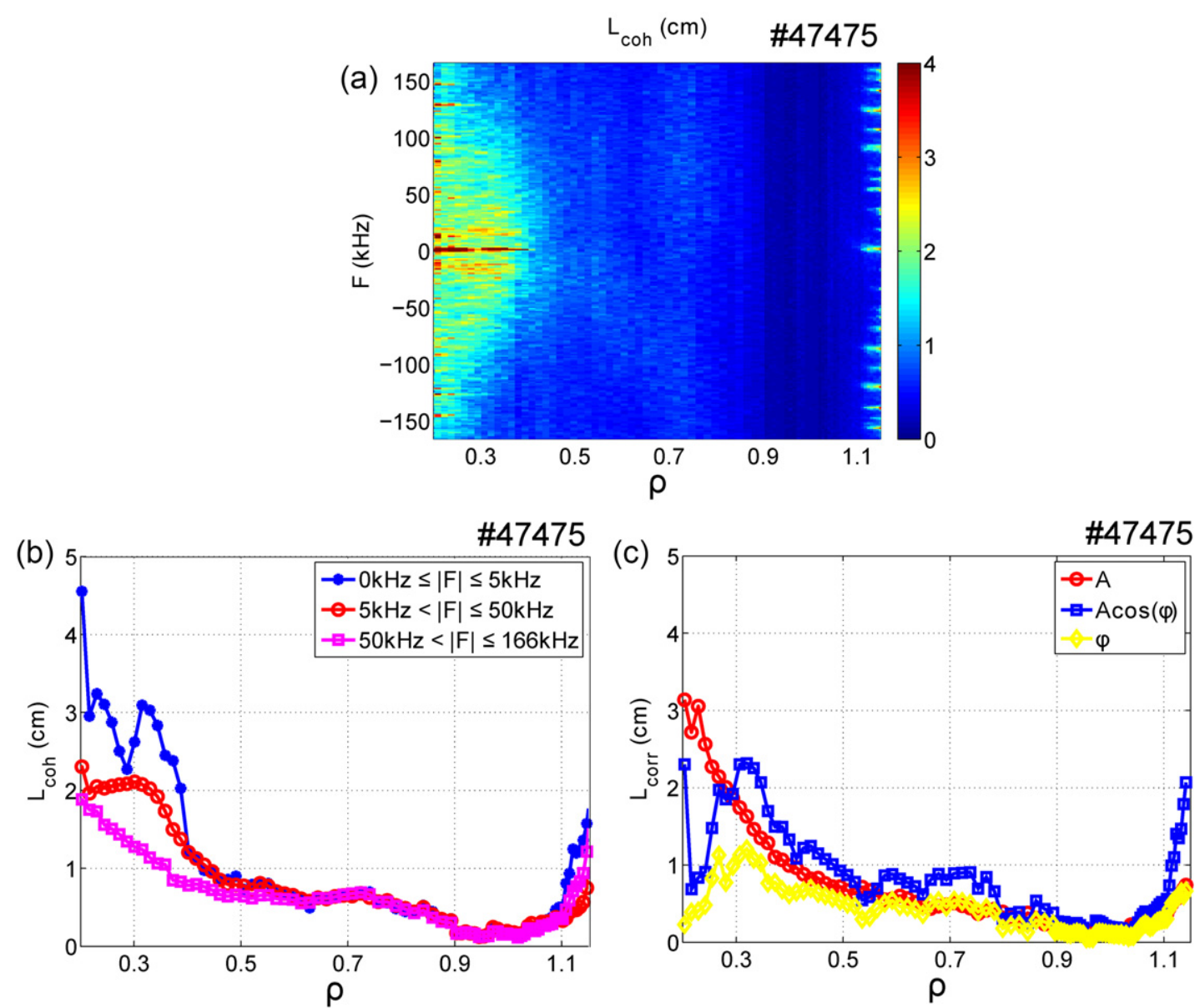

Figure 6. (a) Coherence lengths with respect to the signal frequency and radial position. (b) Radial profiles of the coherence lengths averaged on three distinct frequencies intervals. $(c)$ Radial profiles of the correlation lengths computed on the amplitude, real part and phase signal.

of plasma. Using the measured density profile it was calculated that $40 \mathrm{MHz}$ would correspond to a phase shift ranging approximately up to 2 and $2.4 \mathrm{rad}$ (depending on the density gradient). This explains the measured $\sigma_{\phi}$. Nonetheless, the correct evolution of the phase can still be tracked as shown in figure $10(b)$. Despite the fact that the fixed reflectometer provides a higher antenna directivity $(38 \mathrm{~dB})$, relatively to the swept one $(30 \mathrm{~dB})$, the spectra are comparable (figure $10(c)$ and the fast decrease above $160 \mathrm{kHz}$ observed on the fixed frequency spectra prevents from a possible aliasing disturbance on the swept reflectometer signal.

The ACF measured on the sweeping reflectometer signal drops very quickly to the noise level. Figure $10(d)$ shows a similar behaviour also for the ACF measured with the fixed frequency system which presents only three experimental points before reaching the noise level, leading to a correlation time $t_{\text {corr }}=2.3 \mu \mathrm{s}$. This comparison suggests that the Diraclike ACF measured by ultrafast sweeping reflectometry does not result from a white noise signal but from an insufficient time resolution. Note that, contrary to the core plasma region in presence of MHD activity, the characteristic fluctuation time scale in the edge and SOL region is much shorter than the length of the time series; this stationary turbulence condition justifies the applicability of the cross-correlation technique to evaluate the correlation time and length.
Figure 11(a) shows the radial dependence of the correlation lengths computed on $A, A \cos (\phi)$ and $\phi$. The turbulence must be locally frozen to measure reliable correlation lengths. A plasma region of $40 \mathrm{~cm}$ of radial extent is swept in $2 \mu \mathrm{s}$, the largest structures are therefore swept in $0.1 \mu \mathrm{s}$ which is much shorter than the local correlation time.

As previously observed, the amplitude, phase and real signal correlation lengths show similar behaviour as they decrease from the edge to the LCFS. It is worth noting that at $\rho=0.95$, where the correlation length is minimal, the CCF measured by the sweeping reflectometry is still resolved, as illustrated in figure $11(b)$.

The amplitude, real and phase correlation lengths rapidly decrease above $\rho=0.8$ up to $\rho \approx 0.9$. The drop of the correlation length might originate from two distinct effects. On one hand, the estimated correlation lengths can be artificially reduced due to the high fluctuation level in the edge plasma (figure 9). One the other hand, velocity shear can tear apart the turbulent structures, thus leading to a decrease of the correlation lengths. The perpendicular velocity measured by Doppler reflectometry is shown in figure 12. For $0.85<\rho<$ 0.95 , the plasma rotates in the electron diamagnetic direction with a velocity of the order of $4 \mathrm{~km} \mathrm{~s}^{-1}$. Around $\rho \approx 0.95$, the perpendicular velocity drops by more than a factor two leading to a strong velocity shear. The location of the strong 


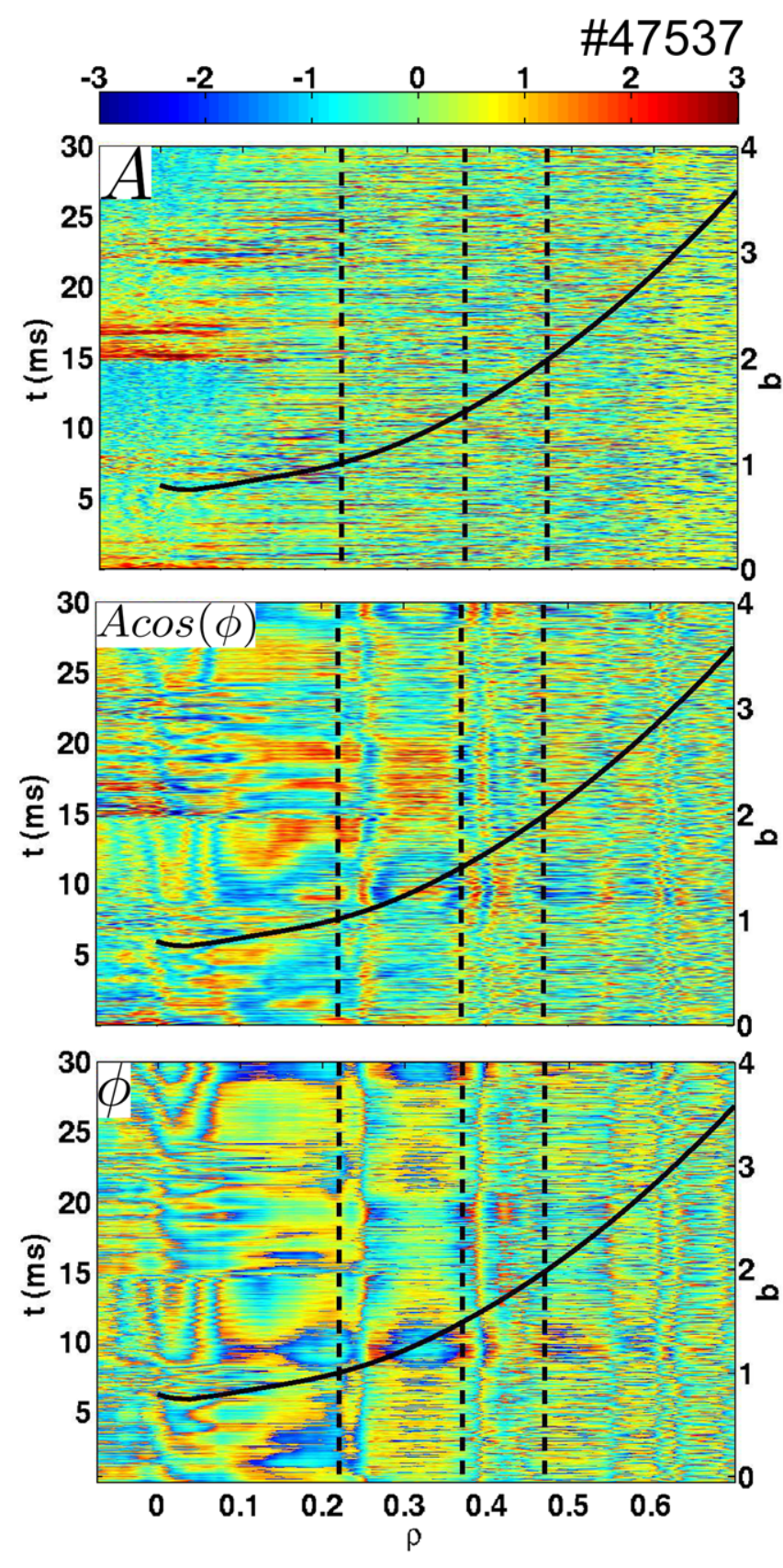

Figure 7. Normalized amplitude (top), real (middle) and phase (bottom) signals with respect to the normalized radial position and time in the core region. The $q$ profile (black line) is also represented. The positions of $q=1,3 / 2$ and 2 rational surfaces are indicated by dashed-lines.

velocity shear is not consistent with the drop of the correlation length. Consequently, the velocity shear might have only little influence on the radial decrease of correlation lengths, the latter is most probably due to an increase of the fluctuation level.

As mentioned above (section 4.1), propagating structures in the SOL are better seen on the amplitude signal thus only this signal is used to characterize the fluctuation in the SOL. The correlation time is lower than $6 \mu$ s close to the LCFS and grows almost exponentially between $d_{\mathrm{LCFS}}=3$ to $7 \mathrm{~cm}$ (figure 13). Further outwards, $t_{\text {corr }}$ stays almost constant with values around $100 \mu \mathrm{s}$.

Figure 14 depicts the radial dependence of the correlation length of the amplitude signal, which increases from about $2 \mathrm{~mm}$ at $d_{\mathrm{LCFS}}=2 \mathrm{~cm}$ to around $12 \mathrm{~mm}$ in the far SOL. At $6 \mathrm{~cm}$ from the LCFS a rapid increase of the correlation length is clearly identified and quantified.

The radial velocity was evaluated in the far SOL as shown in figure 15. Closer to the LCFS, the time lag for which the CCF peaks is zero, therefore the radial velocity cannot be properly inferred in this region with the cross-correlation method. The radial velocity decreases rapidly from 6 to $7 \mathrm{~cm}$ away from the LCFS. Further outwards, the radial velocity decreases only slightly with values between $150 \mathrm{~m} \mathrm{~s}^{-1}$ and $220 \mathrm{~m} \mathrm{~s}^{-1}$.

\section{Summary and discussion}

In this work, the correlation of reflected signals from FMCW reflectometry has been measured on Tore Supra ohmic discharges. The X-mode polarization enabled to extend continuously the measurements from the plasma centre up to the far SOL. It has been here evidenced that turbulence exhibits different characteristics, quantified in terms of correlation lengths, times and propagation velocity, depending on its radial location and that FMCW reflectometry systems can provide fast and ubiquitous information, which is of great help for the data interpretation.

Correlations have been evaluated on each component of the reflectometer signal, amplitude, phase and real part, socalled 'homodyne' signal, as well as the full complex signal, as those account for different plasma fluctuation properties. The question of which reflectometer signal performs the more reliably the turbulence correlation length is still debated. Previous experimental and numerical studies have addressed this issue. By comparing Langmuir probes and reflectometer data measured on CCT tokamak [22] and T10 [23] in presence of strong fluctuations, a close correspondence was obtained between measures on the $A \cos (\phi)$ or complex signals and the ion saturation current. Numerically, turbulent cut-off layers have been modelled by broadband random fluctuations. In the first attempts, the wave-plasma interaction was described with simplified models, namely WKB approximation [15] and physical optics [16]. It was found that the $A \cos (\phi)$ signal better estimates the turbulence correlation length than the phase or the amplitude signals. More recently, a 2D fullwave code was used to simulate O-mode reflectometry [24]. The amplitude correlation length was there found to be in good agreement with the turbulence correlation length for low fluctuation levels (linear regime). At higher fluctuation levels, $L_{\text {corr }}$ appeared to be better estimated from the $A \cos (\phi)$ signal. Overall, the numerical studies have reported that the correlation lengths obtained from the reflectometer signals tend to underestimate the turbulence correlation length at high fluctuation level.

In all the plasma discharges we studied, the measured correlation length is observed to decrease with increasing radius, with values ranging from few centimetres in the core region down to few millimetres towards the LCFS. All the 

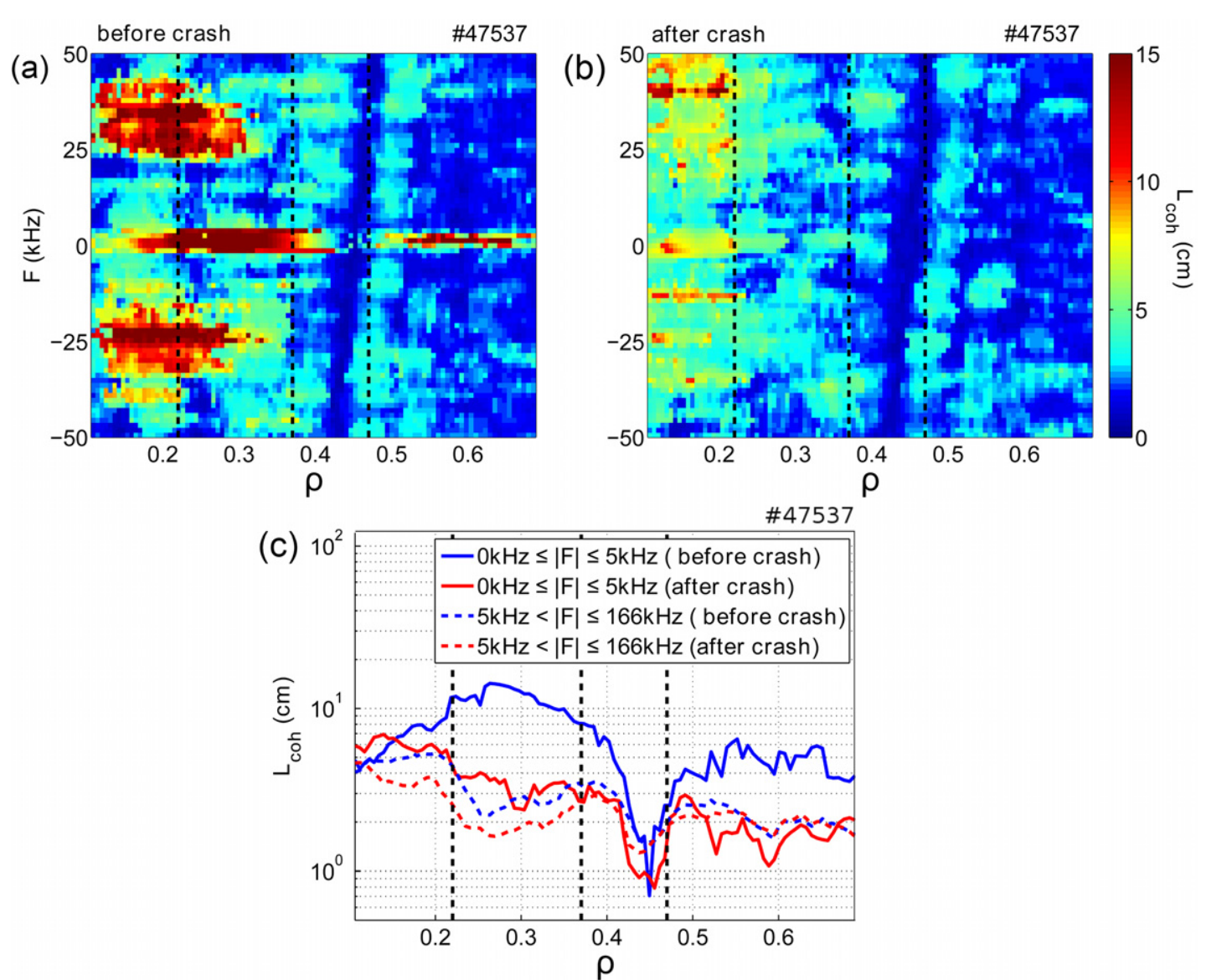

Figure 8. Coherence lengths with respect to the signal frequency and radial position computed before $(a)$ and after the sawtooth crash $(b)$ for the discharge \#47537. (c) Radial profiles of the coherence length computed before (blue curves) and after (red curves) the sawtooth crash for two distinct frequency intervals. The positions of the rational surfaces $q=1,3 / 2$ and 2 are also shown (vertical dashed lines).

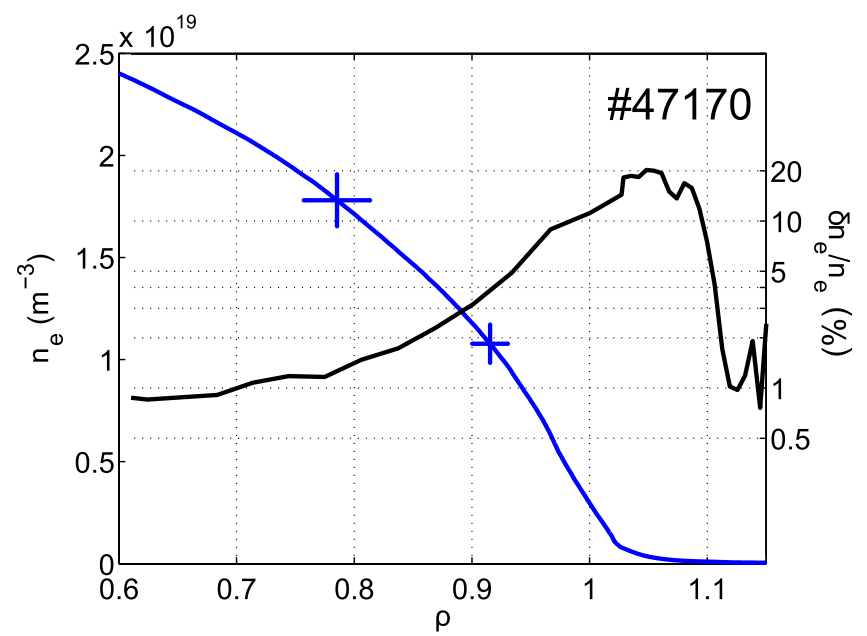

Figure 9. Mean electron density around $t=5 \mathrm{~s}$. The fluctuation level is superposed in full black line. The uncertainties, quantified by the standard deviation, are shown for two radial positions.

signal components present the same trend but quantitatively different values, except close to the LCFS where those do not differ much. In the surroundings of the LCFS, our measured correlation lengths might though be artificially reduced as a consequence of high fluctuation level (see figure 9), coherently to the numerical predictions. We observe nonetheless that the radial dependence and values of the correlation length here measured by ultrafast sweeping reflectometry are very similar to those obtained on ASDEX Upgrade with Doppler reflectometry [25] but contradict the beam emission spectroscopy (BES) measurements on DIII-D which exhibit correlation length in the centimetre range at the plasma edge [26].

In the plasma centre, where the fluctuation level is low $(\delta n / n<1 \%)$, the determination of the correlation length can also be challenging. Thanks to the fast sweep technique, MHD tearing modes, often particularly active in this region, are clearly identified and their radial extension is accessible. A coherency analysis supplies the coherence length profile for low frequencies which overcomes that of microturbulence. This observation indeed provides a better understanding that the correlation lengths can be dominated by these modes rather than by the microturbulence.

The correlation time was estimated in the edge plasma, around 2 to $3 \mu \mathrm{s}$ with the fixed frequency reflectometer measurements and is too short to be effectively measured by our ultrafast sweeping reflectometer. The correlation time measures how long the signature of the turbulence on the reflectometer signals remains unchanged. If we assume that a small variation on the perturbed cut-off layer produces a small variation on the recorded signals, then the respective fluctuation time scales are similar. In the edge plasma, as 

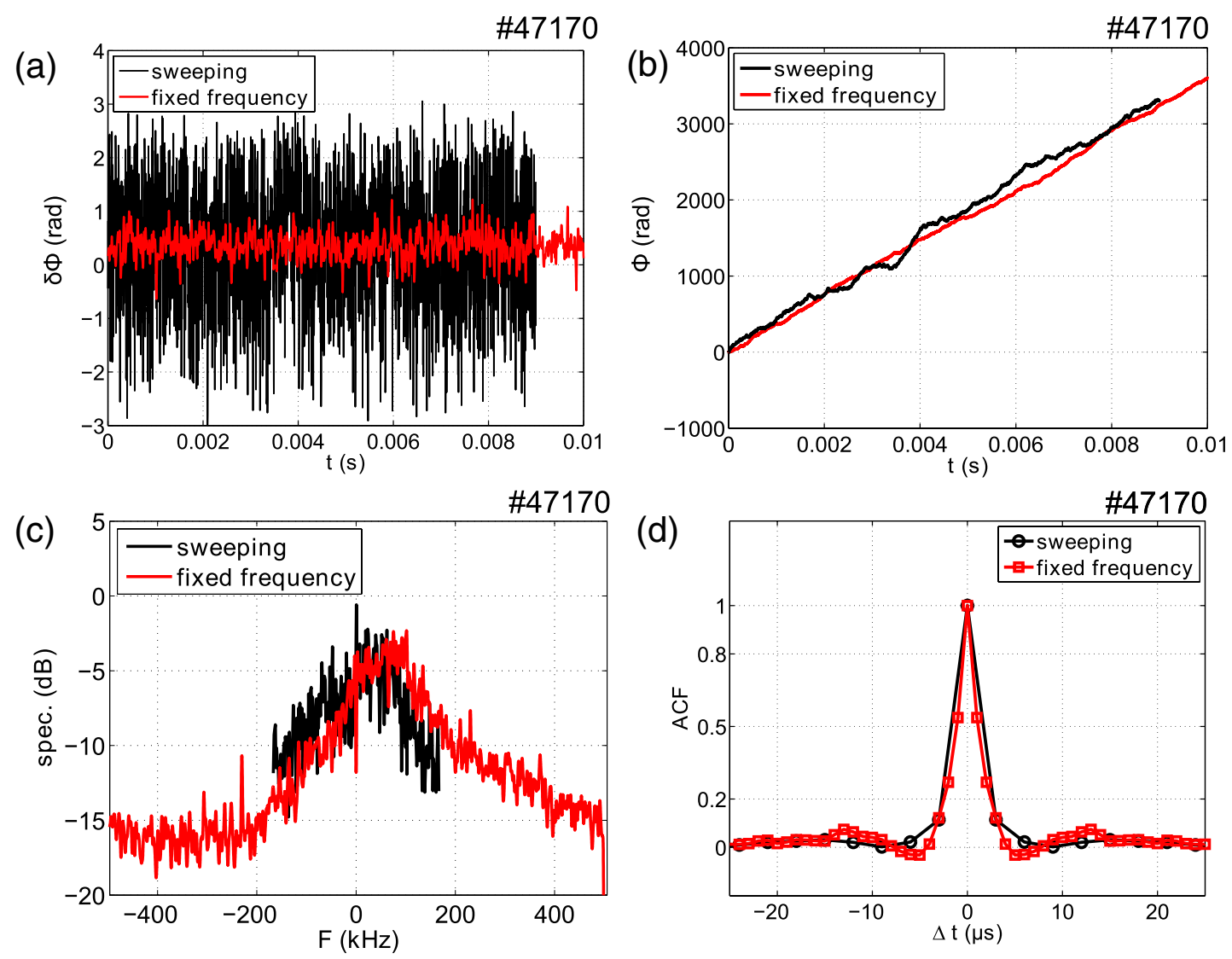

Figure 10. Comparison of the swept ( $3 \mu$ s sampling time) and fixed ( $1 \mu$ s sampling time) frequency reflectometers data measured at $\rho=0.64$ at $t=7.5 \mathrm{~s}$. (a) phase and $(b)$ phase fluctuation time history, $(c)$ power spectra and $(d)$ amplitude ACF.
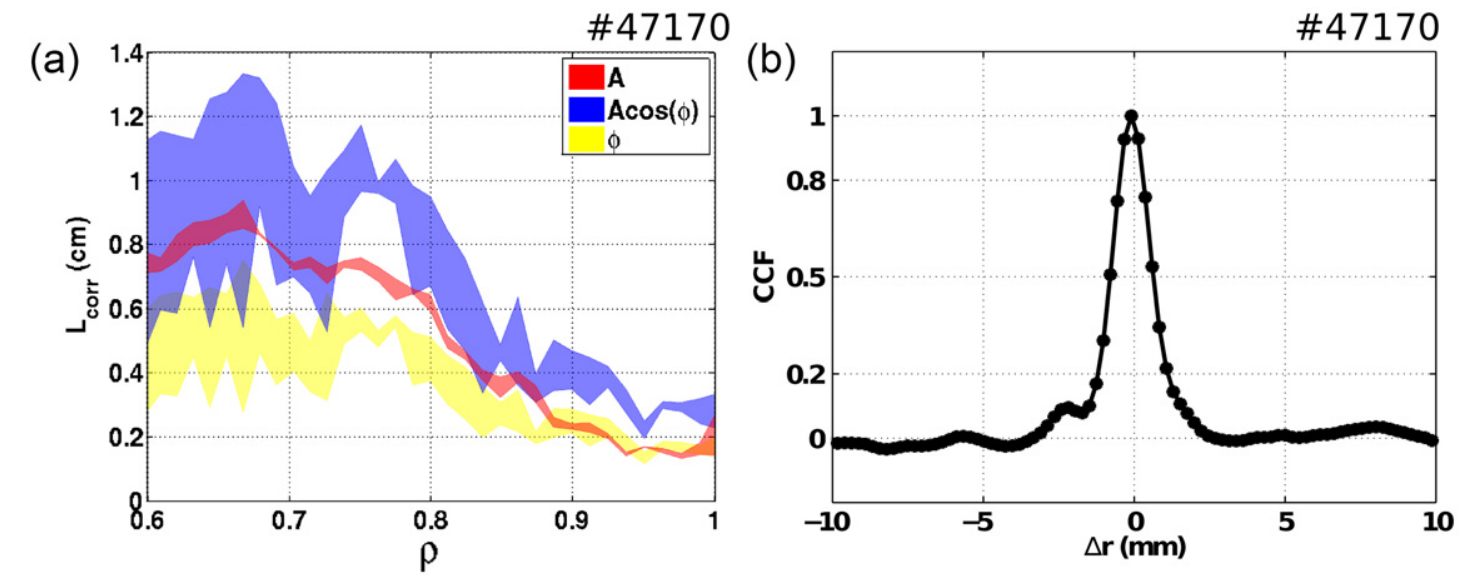

Figure 11. (a) Correlation lengths computed in the edge region on the amplitude, real and phase signal. For each signal, the shaded area in between the maximum and minimum correlation length represents the dispersion of the results. (b) Amplitude CCF computed at $\rho=0.95$ around $t=5 \mathrm{~s}$.

the poloidal velocity is dominant with respect to the radial velocity, the correlation time should approximately scale as $t_{\text {corr }} \approx L_{\mathrm{c}} / V_{\theta}$, where $L_{\mathrm{c}}$ is the turbulence characteristic length. Assuming that turbulence structures have circular shapes in the $r-\theta$ plane [27], $L_{\mathrm{c}} \approx L_{\theta} \approx L_{r}$, and using the calculated correlation lengths ranging within $1 \mathrm{~cm}$ and $1 \mathrm{~mm}$ the poloidal velocity should range between few thousands to few hundreds $\mathrm{m} \mathrm{s}^{-1}$. This estimate is coherent with Doppler reflectometry measurements on Tore Supra [28]. Large scale propagating structures in the far SOL have been observed for the first time using ultrafast sweeping reflectometry. In the case of SOL-like turbulence [29], we have considered the amplitude signal to be the most appropriate to estimate the turbulence correlation characteristics, as coherent structures and their dynamics are there better defined. The correlation time is found to increase radially towards the far SOL, in agreement with measurements performed with Langmuir probes on Tore Supra [30]. While the shape of these turbulent structures can play a significant role 


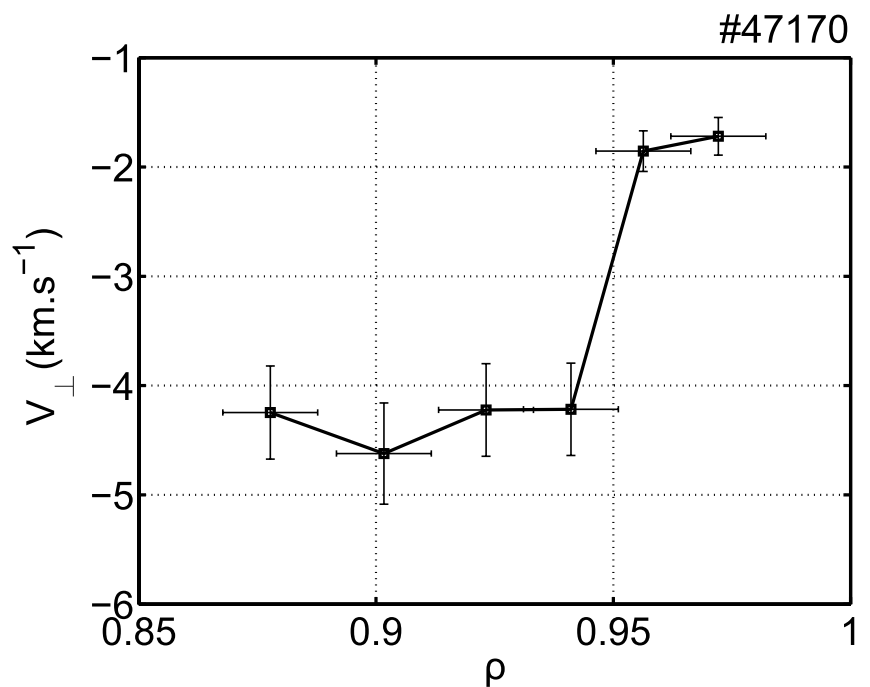

Figure 12. Radial profile of the perpendicular velocity measured by Doppler reflectometry.

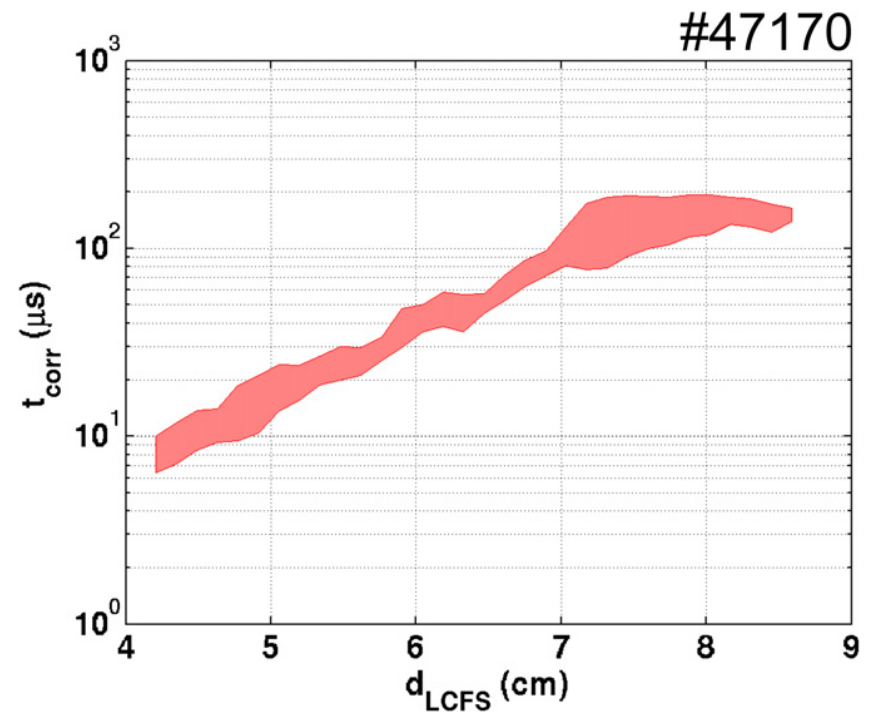

Figure 13. Amplitude correlation time in the SOL with respect to the distance to the LCFS $\left(d_{\mathrm{LCFS}}\right)$. The shaded area, in between the maximum and minimum correlation time, represents the variation of the results during the three bursts of sweeps.

when determining velocities from cross-correlation analysis [31] our calculation gives an estimate of $\sim 150-200 \mathrm{~m} \mathrm{~s}^{-1}$ for their radial velocity.

Much effort has been devoted to model reflectometry numerically. Most of the models have used broadband turbulence so far. In the light of the new results obtained with ultrafast sweeping reflectometry in Tore Supra SOL, attempts to study numerically the behaviour of reflectometry in SOL turbulence conditions would be of a great interest.

\section{Acknowledgments}

Fruitful discussions with Professor Heuraux, Drs Fedorczak and Tamain are gratefully acknowledged. This work, supported by the European Communities under the contract of Association between EURATOM and CEA, was carried out

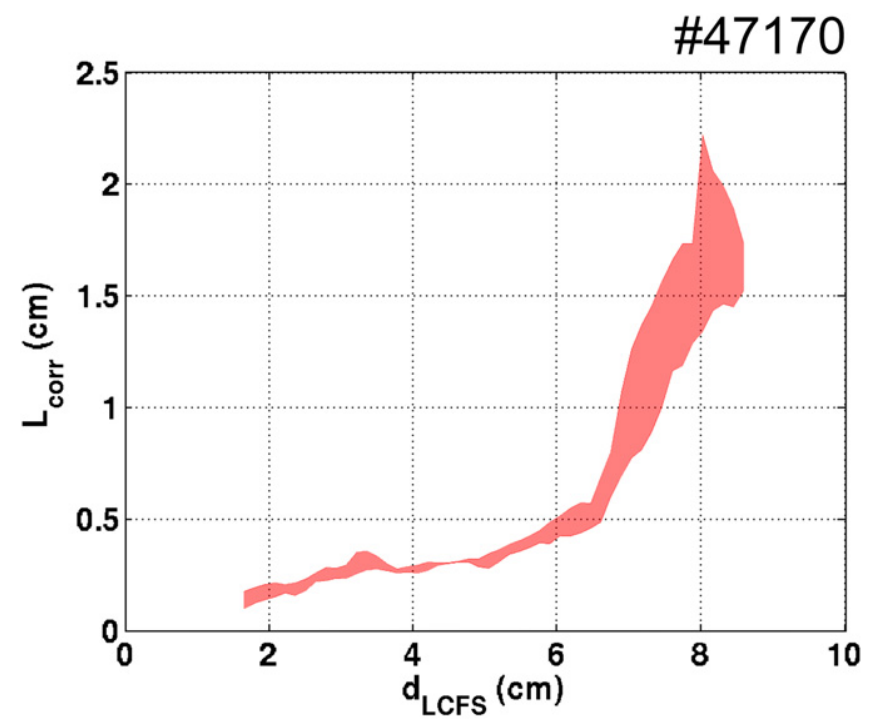

Figure 14. Amplitude correlation length in the SOL with respect to the distance to the LCFS $\left(d_{\mathrm{LCFS}}\right)$. The shaded area in between maximum and minimum the correlation length represents the variation of the results during the three bursts of sweeps.

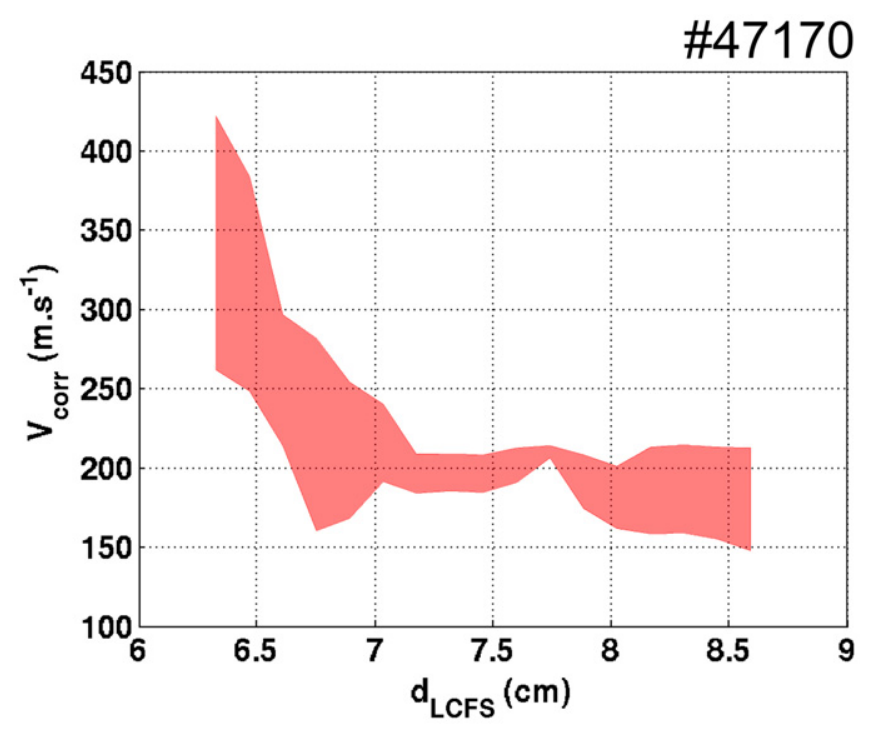

Figure 15. Radial velocity evaluated on the amplitude signal with respect to the distance to the LCFS $\left(d_{\mathrm{LCFS}}\right)$. The shaded area in between the maximum and minimum radial velocity represents the variation of the results.

within the framework of the European Fusion Development Agreement. The views and opinions expressed herein do not necessarily reflect those of the European Commission.

Euratom @ 2013.

\section{References}

[1] Hanson G R, Wilgen J B, Anabitarte E, Bell J D, Harris J H, Dunlap J L and Thomas C E 1990 Rev. Sci. Instrum. $613049-51$

[2] Rhodes T L, Peebles W A and Doyle E J 1992 Rev. Sci. Instrum. 63 4661-4

[3] Sanchez J, Branas B, Delaluna E and Estrada T 1993 Rev. Sci. Instrum. 64 487-91 
[4] Mazzucato E and Nazikian R 1993 Phys. Rev. Lett. 71 1840-3

[5] Nazikian R et al 2005 Phys. Rev. Lett. 94135002

[6] Urazbaev A O, Vershkov V A, Soldatov S V and Shelukhin D A 2006 Plasma Phys. Rep. 32 443-60

[7] Kraemer-Flecken A, Soldatov S, Vowinkel B and Mueller P 2010 Rev. Sci. Instrum. 81113502

[8] Kosolapova N V, Gusakov E Z and Heuraux S 2012 Plasma Phys. Control. Fusion 54035008

[9] Kurzan B, Hempel S D, Holzhauer E, Scott B, Serra F, Suttrop W, Zeiler A and the ASDEX Upgrade Team 2000 Plasma Phys. Control. Fusion 42 237-53

[10] Clairet F, Heuraux S, Bottereau C, Molina D, Ducobu L, Leroux F and Barbuti A 2010 Rev. Sci. Instrum. 81100903

[11] Clairet F, Sabot R, Bottereau C, Chareau J M, Paume M, Heuraux S, Colin M, Hacquin S and Leclert G 2001 Rev. Sci. Instrum. 72 340-3

[12] Sabot R, Sirinelli A, Chareau J M and Giaccalone J C 2006 Nucl. Fusion 46 S685-92

[13] Bottollier-Curtet H and Ichtchenko G 1987 Rev. Sci. Instrum. 58 539-46

[14] Clairet F, Bottereau C, Chareau J M and Sabot R 2003 Rev. Sci. Instrum. 74 1481-4

[15] Estrada T, Sanchez J, Zhuravlev V, de la Luna E and Branas B 2001 Phys. Plasmas 8 2657-65

[16] Conway G D 1997 Plasma Phys. Control. Fusion 39 407-21

[17] Vermare L, Heuraux S, Clairet F, Leclert G and da Silva F 2006 Nucl. Fusion 46 S743-59

[18] Vermare L, Clairet F, Heuraux S and Leclert G 2005 Plasma Phys. Control. Fusion 47 1895-909
[19] Sabot R et al 2006 Plasma Phys. Control. Fusion 48 B421-32

[20] Artaud J F et al 2010 Nucl. Fusion 50043001

[21] Maget P, Artaud J F, Eriksson L G, Huysmans G, Lazaros A, Moreau P, Ottaviani M, Segui J L and Zwingmann W 2005 Plasma Phys. Control. Fusion 47 357-77

[22] Rhodes T L, Peebles W A, Doyle E J, Pribyl P, Gilmore M, Moyer R A and Lehmer R D 1998 Plasma Phys. Control. Fusion 40 493-510

[23] Urazbaev A O, Vershkov V A, Soldatov S V and Shelukhin D A 2006 Plasma Phys. Rep. 32 619-41

[24] Blanco E, Estrada T and Happel T 2009 Proc. 9th Int. Reflectometry Workshop - IRW9 (Lisboa, May 2009)

[25] Schirmer J, Conway G D, Holzhauer E, Suttrop W, Zohm H and the ASDEX Upgrade Team 2007 Plasma Phys. Control. Fusion 49 1019-39

[26] Shafer M W, Fonck R J, McKee G R, Holland C, White A E and Schlossberg D J 2012 Phys. Plasmas 19032504

[27] Mckee G R, Fenzi C and Fonck R J 2002 IEEE Trans. Plasma Sci. 30 (Part 1) 62-3

[28] Hennequin P, Honore C, Truc A, Quemeneur A, Fenzi-Bonizec C, Bourdelle C, Garbet X, Hoang G T and Tore Supra Team 2006 Nucl. Fusion 46 S771-9

[29] D'Ippolito D A, Myra J R and Zweben S J 2011 Phys. Plasmas 18060501

[30] Nanobashvili I, Gunn J P and Devynck P 2007 J. Nucl. Matter. 363-365 622-7

[31] Fedorczak N, Manz P, Thakur S C, Xu M, Tynan G R, Xu G S and Liu S C 2012 Phys. Plasmas 19122302 